\title{
O papel da experiência de compra na intenção de recompra
}

\author{
The role of purchase experience on repurchase intention
}

\author{
Carlos Alberto Alves ${ }^{1}$ \\ Claudio José Stefanini ${ }^{2}$ \\ Leonardo Aureliano da Silva ${ }^{3}$ \\ Sérgio Luiz do Amaral Moretti ${ }^{4}$
}

\begin{abstract}
Resumo
O propósito deste trabalho é investigar a relação entre a experiência de compra e a intenção de recompra, e o papel mediador da satisfação e da confiança do consumidor nesta relação. A hipótese central do trabalho prevê que a experiência de compra possui relação positiva e significante com a intenção de recompra. Foi realizada uma pesquisa tipo survey com 416 consumidores de São Paulo. Os resultados apontam que os consumidores cujas experiências de compra são positivas tendem a possuir intenções de recompra com o mesmo varejista de forma positiva. Como contribuição gerencial, este trabalho mostra a importância do varejista em construir pistas que aumentem a experiência de compra do consumidor, como a atmosfera da loja, incentivo à participação de companhias durante o período de compra e a criação de laços de afeição entre o consumidor e a loja. Sendo assim, a pesquisa se justifica tanto teoricamente como gerencialmente.
\end{abstract}

Palavras-chave: Marketing; Varejo; Intenção de recompra; Experiência de compra.

\begin{abstract}
The purpose of this study is to investigate the relationship between the shopping experience and the repurchase intention and the mediating role of satisfaction and consumer trust in this relationship. The central hypothesis of the study predicts that the shopping experience has a positive and significant relationship with the repurchase intention. research type survey with 416 consumers in São Paulo. The results show that consumers whose shopping experiences are positive tend to have repurchase intentions with the same retailer positively. As a managerial contribution, this work shows the importance of the retailer to build cues that enhance the consumer shopping experience, as the atmosphere of the store, encouraging the participation of companies during the purchase period and creating bonds of affection between the consumer and the store. Thus, the research is justified both theoretically and managerially.
\end{abstract}

Keywords: Marketing; Retail; Repurchase intention; Purchase experience.

\section{Introdução}

Experiências possuem um papel crítico no contexto de compras e consumo. Pessoas compram para adquirir produtos, obter informações de produtos, ou pelo prazer da atividade em si (Tauber, 1972). Além do valor utilitário embutido na aquisição dos produtos, o ato de comprar também fornece valor hedônico ao consumidor. Nesse sentido, alguns autores (Meyer \& Schwager, 2007; Voss, 2003) apontaram que a experiência de compra pode fornecer novos meios de competição. Fazer com que o consumidor tenha uma boa experiência de compra é importante, pois pode afetar a satisfação (Rawson, Duncan, \& Jones, 2013) ea lealdade (Berry \& Carbone, 2007; Pullman \& Gross, 2004), influenciar as expectativas e incutir confiança no consumidor (Flanagan, Johnston, \& Talbot, 2005).

\footnotetext{
Doutor em Administração pela Universidade Nove de Julho - UNINOVE. Brasil. Afiliação: Universidade Anhembi Morumbi (UAM). Lattes: http:// lattes.cnpq.br/9085019105914550ORCID: http://orcid.org/0000-0003-2260-5580Email: calves761@gmail.com

2 Doutor em Administração pela Fundação Educacional Inaciana Padre Sabóia de Medeiro (FEI). Brasil. Afiliação: Universidade Anhembi Morumbi (UAM). Lattes: http://lattes.cnpq.br/1728750139990470Email: cjstefanini@gmail.com

3 Doutor em Administração pela Universidade Nove de Julho - UNINOVE. Brasil. Afiliação: Universidade Anhembi Morumbi (UAM). Lattes: http:// lattes.cnpq.br/2136195205924034Email: leonardoaureliano@outlook.com

4 Doutor em Ciências Sociais pela Pontifícia Universidade Católica de São Paulo, PUC/SP. Brasil. Afiliação: Universidade Anhembi Morumbi (UAM). Lattes: http://lattes.cnpq.br/1686957518540720Email: sergiomoretti@uol.com.br
} 
A experiência de compra é importante, porque também é um antecessor da intenção de retornar ao estabelecimento varejista e repetir a experiência de compra. Assim, não são poucos os estudos que consideram a intenção de recompra (Alves, 2016; Julander \& Söderlund, 2003; Mittal, Ross, \& Baldasare, 1998).

Portanto, com base nos argumentos apresentados, considera-se importante um estudo empírico que investigue essa temática. Dessa forma, coloca-se a seguinte questão de pesquisa: Qual a relação entre a experiência de compra e a intenção de recompra, mediadas pela satisfação e pela confiança? O objetivo geral deste trabalho é investigar a relação entre a experiência de compra e a intenção de recompra, mediadas pela satisfação e pela confiança do consumidor. Como objetivos específicos, podemos enumerar: primeiro, compreender o fenômeno experiência de compra; segundo, aprofundar o entendimento dos construtos satisfação, confiança e intenção de recompra.

Este trabalho se justifica pelo entendimento de conhecimentos sobre a experiência de compra e seus constituintes e sua relação com a intenção comportamental, além da interação mediadora de dois importantes construtos discutidos na literatura, a satisfação e a confiança.

\section{Referencial teórico}

\subsection{Experiência de compra}

A definição para experiência de compra é descrita como as respostas internas e subjetivas que o consumidor possui para qualquer contato direto e indireto com a loja varejista (Meyer \& Schwager, 2007). Por sua vez, Gentile, Spiller e Noci (2007) definem a experiência de compra como aquela originada de um conjunto de interações entre o consumidor e um produto, uma loja, ou uma parte da organização, as quais provocam uma reação. Dessa forma, o conceito em si é um construto psicológico que, de forma holística, é resultante das respostas subjetivas do contato direto e indireto do consumidor com o varejista,abrangendo diferentes níveis de envolvimento (Gentile, Spiller, \& Noci, 2007; Johnston \& Kong, 2011; Lemke, Clark, \& Wilson, 2011, Stefanini, Alves \& Marques, 2018). Segundo Gentile, Spiller e Noci (2007), existem seis níveis de envolvimento na experiência de compra: sensorial, emocional, cognitivo, pragmático, estilo de vida e racional. Para Lemke, Clark e Wilson (2011), a experiência é estritamente pessoal e implica no envolvimento do consumidor em vários níveis (racional, emocional, sensorial, físico e espiritual). Portanto, a experiência de compra incorpora o total de experiências de compra, tão discutido na literatura sobre o comportamento do consumidor, incluindo a procura, a decisão, a compra, o consumo e o pós-consumo (Verhoef et al., 2009).

As primeiras caracterizações e conceitualizações da experiência de compra sugeriam que essas experiências, dentro de um ambiente de varejo, podiam ser descritas em quatro dimensões: alegria, humor, lazer e únicas (Bagdare \& Jain, 2013). Tais dimensões, porém, são limitadas, no sentido de que não abrangem outras perspectivas discutidas na literatura. Na abordagem discutida por Rose, Clark, Samouel \& Hair (2012), cognição e afeto foram consistentemente identificados como componentes influenciadores do comportamento do consumidor e da experiência de compra (Frow\& Payne, 2007). Por sua vez, a abordagem social mostra que os grupos de referência são influenciadores do comportamento do consumidor (Haytko \& Baker, 2004; Luo, 2005; White \& Dahl, 2006) e estão incorporados em modelos de experiência de compra como os discutidos por Verhoef et al. (2009). No mesmo sentido, a atmosfera da loja é identificada na literatura como um dos componentes da experiência de compra.

A cognição consiste nas crenças, pensamentos ou percepções formadas por meio de interações diretas com as ofertas (bens, serviços e ambientes de compra) feitas ao consumidor, do processamento de fontes de informações secundárias (boca a boca, blogs, propagandas/publicidades, comentários de produtos etc.) (Bagdare \& Jain, 2013) e também da comparação de informações contra os esquemas cognitivos representativos e a memória (Bagdare \& Jain, 2013; Holbrook, 1986). Além disso, dicas ou pistas no ambiente de loja podem estimular a atividade mental, representada pelo número de pensamentos gerados pelo encontro de vendas e a profundidade do processamento de informações por parte do consumidor (Chebat, Chebat, \& Vaillant, 2001), assim como pelo total de vezes gasto pelo consumidor no processamento de informações sobre os estímulos (nome da marca) encontrados no ambiente (Morrin \& Ratneshwar, 2000). 
O termo emoção pode ser empregado como um guarda-chuva para um conjunto mais específico de processos mentais, como humor, afeição e, possivelmente, atitudes (Bagozzi, Gopinath \& Nyer, 1999; A. R. Johnson \& Stewart, 2005). Portanto, emoção pode ser considerada mais como categoria geral para um processo mental dos sentimentos do que um processo psicológico específico. Por sua vez, emoção é o estado de prontidão que surge da avaliação cognitiva dos eventos ou pensamentos. Possui um tom fenomenológico e é acompanhada por um processo fisiológico frequentemente expresso fisicamente por: gestos, posturas, características faciais, e pode resultar em ações específicas para afirmar ou lidar com as emoções, dependendo da natureza e do significado para a pessoa que a está sentindo (Bagozziet al., 1999; A. R. Johnson \& Stewart, 2005).

Comprar, inerentemente, é uma experiência social. Companhias podem melhorar a experiência de compra compartilhando as experiências e dando suporte e assistência na tomada de decisão (Borges, Chebat\&Babin, 2010; Lindsey-Mullikin \& Munger, 2011). De forma consistente com essa visão, pesquisas em Marketing sugerem que comprar com uma companhia leva a resultados positivos. Da mesma forma, a presença de uma companhia durante o processo de compra reduz o risco percebido e aumenta a confiança do comprador na decisão realizada (Borges et al., 2010; Kiecker \& Hartman, 1994). Uma companhia pode aumentar as emoções associadas a compras(Matzler, Faullant, Renzl, \& Leiter, 2005) e cria uma experiência de compra mais hedonicamente orientada (Kesari, \& Atulkar, 2016), além de encorajar trocas sociais com outras pessoas (Goby, 2006).

O ambiente da loja possui diversos estímulos que podem ser percebidos pelos sentidos dos compradores. Dessa forma, a atmosfera de uma loja pode interferir na experiência de compra, influenciando o humor de um comprador por meio da excitação e prazer (Mehrabian \& Russell, 1974). Assim, o ambiente de compras pode ser projetado com o propósito específico de produzir efeitos sensoriais e emocionais nos compradores, e aumentar a probabilidade de realizarem uma compra (Ballantine, Jack, \& Parsons, 2010).

Exemplos de respostas dos consumidores induzidas por mudanças na variável atmosfera do ambiente incluem vendas devido a arranjos em vitrines exteriores de lojas (Mower, Kim, \& Childs, 2012), o efeito da iluminação e o número de itens manuseados pelos compradores (Mohan, Sivakumaran, \& Sharma, 2013), o efeito da música em uma loja de roupas (Yalch \& Spangenberg, 2000), o layout da loja na percepção de preço (Mohanet al., 2013) e a organização dos elementos de merchandising (Feijó \& Botelho, 2012; Law, Wong, \& Yip, 2012). Elementos como música, cores, odores, temperatura, layout e iluminação, e suas possíveis combinações, constituem a atmosfera de uma loja (Yalch \& Spangenberg, 2000).

A literatura aponta três resultados comportamentais para a experiência de compra: satisfação, confiança e intenção de recompra (Janda \& Ybarra, 2005).

\subsection{Satisfação, confiança e intenção de recompra}

Satisfação do consumidor com um determinado produto foi proposto por Cardozo (1965). Satisfação é o resultado de uma avaliação pós-compra e é uma resposta afetiva, de forma geral, da experiência de compra (Bagozzi et al., 1999; Oliver, 1980, Alves, 2017). Dessa forma, as emoções, que são componentes importantes do afeto, são conhecidas por estarem associadas com intensos estados de prazer,os quais levam a uma atenção focada em objetivos específicos e podem, consequentemente, impactar no comportamento.

Segundo Athiyaman (2004), as teorias sobre satisfação do consumidor podem, de forma geral, serem classificadas em três grandes grupos:Desconfirmação da expectativa, Equidade e Atribuição.

Confiança é definida como uma disposição em acreditar nas ações de um parceiro, com o qual existe uma relação, baseado na expectativa de que esse parceiro realize uma dada ação sem que exista a necessidade de um monitoramento ou controle sobre a ação realizada (Moorman, Deshpande, \& Zaltman, 1993). A confiança pode ser identificada também como confiança cognitiva e confiança afetiva (Johnson \& Grayson, 2005; Moorman et al., 1993).

A confiança cognitiva, ou aprendida, é a certeza ou disposição em depender da competência e confiabilidade de outra parte (Moorman et al., 1992). 
A confiança afetiva é a intuição, sentimento ou convicção que se coloca na outra parte com base nos sentimentos gerados pelo nível de cuidado e preocupação que a outra parte demonstra (Johnson-George \& Swap, 1982; Rempel et al., 1985).

\subsection{Desenvolvimento das hipóteses e modelo teórico proposto}

Intenção de recompra foi investigada extensivamente por pesquisadores de Marketing e é tipicamente definida como a intenção de comprar repetidamente um produto ou serviço do mesmo fornecedor (Alves, 2016; Hellier, Geursen, Carr, \& Rickard, 2003; Kim, Galliers, Shin, Ryoo, \& Kim, 2012).Zeithaml et al. (1996) sugere dois tipos de intenção de recompra: a primeira é a intenção de recomprar, e a segunda é a intenção em se empenhar em um boca a boca positivo.

Um dos antecedentes da intenção de compra é a experiência de compra do consumidor,segundo Hellier et al (2003). Dessa forma se pode expressar a relação entre a experiência de compra e a intenção de recompra como sendo a primeira hipótese formulada da pesquisa e é proposta como sendo: $\mathrm{H}_{1}$ : Existe uma relação positiva e significante entre a experiência de compra e a intenção de recompra

Diversas pesquisas exploram a relação mediadora da satisfação e da confiança na intenção de recompra do consumidor (Alves, 2017; Mittal \& Kamakura, 2001; Stefanini, et. al., 2017; Stefanini, et. al.,2018; Zboja \& Voorhees, 2006). Uma relação de mediação ou efeito indireto ocorre quando o efeito causal de uma variável independente $\mathrm{X}$ em uma variável dependente $\mathrm{Y}$ é transmitida por um mediador M (Preacher, et al., 2007). Neste sentido, expressa-se a relação mediadora da confiança e da satisfação entre a experiência de compra e a intenção de recompra do consumidor como duas hipóteses de pesquisa, as quais são formuladas como mostradas a seguir: $\mathrm{H}_{2}$ : A Satisfação é uma mediadorada relação entre a experiência de compra e a intenção de recompra; $\mathrm{H}_{3}$ : Confiança é uma mediadora da relação entre a experiência de compra e a intenção de recompra.

Diante do apresentado, estrutura-se o modelo teórico proposto, apresentado na figura 1.

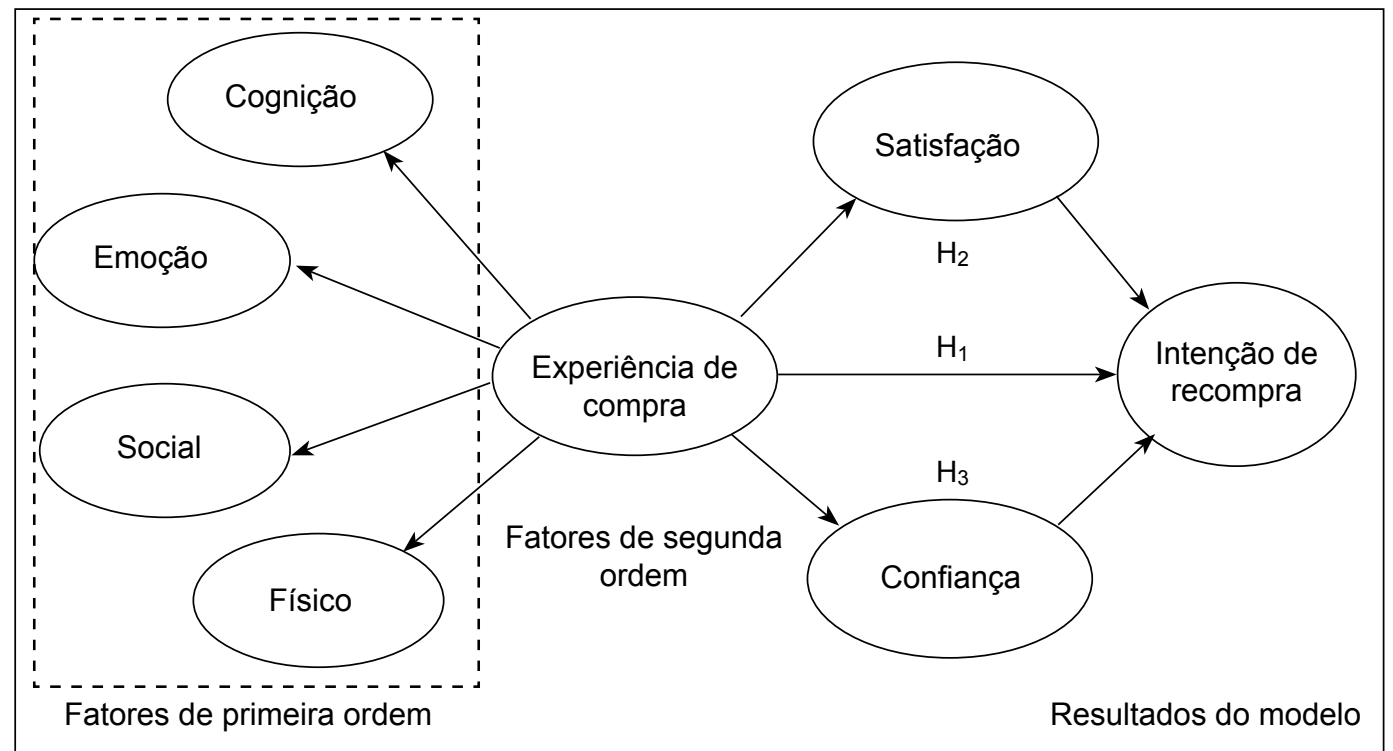

Figura 1 - Modelo estrutural proposto

O modelo estrutural proposto também é uma rede nomológica usada para testar a validade dos construtos das nossas variáveis. Os construtos são considerados nomológicamente válidos se, conforme as mensurações realizadas pela parte empírica deste estudo, prevejam ou sejam preditos por outros construtos consistentes com a revisão da literatura e/ou com pesquisas realizadas anteriormente (Cronbach 1971, Bagozzi 1980). 


\section{Metodologia}

\subsection{Amostra e coleta de dados}

A amostra é não probabilística e por conveniência (Krzanowski, 2007). A escolha por amostra não probabilística se deu devido ao grande número de estabelecimentos varejistas espalhados na cidade de São Paulo e pela dificuldade em se conseguir, aleatoriamente, um contato para a realização da pesquisa. Outros fatores que impactaram na decisão pela amostra não probabilística foram: limitação orçamentária e limitação de tempo. A coleta de dados ocorreu entre os meses de novembro e fevereiro de 2016. O levantamento foi realizado por três equipes de pesquisa, compostas por seis indivíduos cada, perfazendo um total de 18 pessoas. A área pesquisada constituiu-se de centros comerciais de bairro (classificação apresentada por Entenberg (1960)), todos da zona sul da cidade de São Paulo. Foram entrevistados 985 consumidores para entrega do instrumento de pesquisa. Dos entrevistados, 518 aceitaram responder ao instrumento, ou seja, uma taxa de $52,5 \%$. Dos questionários retornados, apenas 416 foram empregados na análise, tendo sido 102 descartados por possuírem algum tipo de erro que inviabilizava seu uso, como falta de resposta em alguns dos itens ou duplo preenchimento, entre outros problemas percebidos.

\subsection{Escalas utilizadas no modelo}

Seguindo as sugestões de Herzog e Bachman (1981), esforços foram feitos para evitar questões ambíguas, prestando-se atenção na sequência de questões e buscando-se um formato e estilo profissionais. As inconsistências e ambiguidades do instrumento de pesquisa foram corrigidas e eliminadas antes do trabalho de campo (Herzog \& Bachman, 1981; Malhotra, 2011).

A mensuração das emoções foi adaptada das escalas desenvolvidas por Havlena e Holbrook (1986) e por Babinet al. (1998), e consistia de cinco itens. A mensuração da cognição foi adaptada da escala desenvolvida por Cacioppo \& Petty (1982) e Oliver (1980), e consistia de seis itens. Companhias do consumidor, durante o processo de compra, podem afetar as decisões de compra, resultando em mais ou menos aquisições. O comprador social é aquele que tem outras pessoas por companhia durante uma visita a uma loja para realização de compras.Por consequinte,os compradores solitários são aqueles que vão às compras sozinhos (Goswami \& Mishra, 2009; Nicholls, 1997).

A atmosfera da loja foi mensurada empregando-se uma combinação das escalas desenvolvidas por Baker, Grewal, e Parasuraman, (1994), Grewal, Baker, Levy, e Voss, (2003), Michon, Chebat e Turley (2005). A confiança foi mensurada por meio de uma escala de quatro itens adaptada de várias fontes (Morgan \& Hunt, 1994; Verhoef, Franses, \& Hoekstra, 2002). A satisfação foi mensurada por meio de uma escala de nove itens adaptada também de várias fontes (Fullerton, 2005; Garbarino\& Johnson, 1999; Grace \& O'Cass, 2005), assim como a intenção de recompra foi mensurada por meio de uma escala de cinco itens adaptada (Fullerton, 2005; M. D. Johnson, Herrmann, \& Huber, 2006).

Em todas as mensurações, os consumidores indicavam sua concordância em uma escala de sete pontos, tipo Likert, ancorada em 1 = Discordo totalmente e 7 = Concordo totalmente.

\subsection{Plano para análise dos dados}

Com o objetivo de testar o modelo teórico, decidiu-se pela utilização da modelagem de equações estruturais (MEE). Hairet al. (2009) indicam que a MEE é a abordagem que fornece os meios necessários para avaliar teorias que são conceitualmente importantes. Como recomenda a literatura (Kline, 2005; Lopes, 2008), verificaram-se, antecipadamente, os pressupostos para a aplicação dessa técnica regressiva, que são os testes de linearidade, multicolinearidade, normalidade e homocedasticidade.

A linearidade foi verificada por meio da matriz de correlações (Hairet al., 2009). A ausência de multicolinearidade foi verificada por meio do cálculo do fator de inflação da variância (VIF). A homocedasticidade foi verificada por meio do cálculo de Levene (Hairet al., 2009) e, finalmente, a normalidade na distribuição das variáveis dependentes do modelo foi verificada por meio do teste de Kolgomorov-Smirnov (Hairet al., 2009; Levin\& Fox, 2006; Lopes, 2008). 
Após os testes preliminares, aplicou-se a MEE seguindo a metodologia proposta por Chin e Newsted (1999), com estimação dos mínimos quadrados parciais (PLS-PM - PartialLeast Square - Path modelling), por meio do software SmartPLS 2.0M3 (Ringle, Wende, \& Will, 2005) e das recomendações de Anderson e Gerbin (1988), com a análise realizada em duas fases. Na primeira fase, analisou-se o modelo de mensuração por meio da verificação das validades convergente e discriminante; na segunda, realizou-se a análise do modelo estrutural (Anderson \&Gerbing, 1988).

Diferente da MEE baseada em covariâncias, o PLS não otimiza uma função global (Bido et al., 2011), por isso não há índices de ajustamento dos modelos (como RMSEA, CFI, NFI, entre outros) identificados por meio de softwares, como o LISREL, o AMOS e o EQS. Por outro lado, Tenenhuaus et al. (2005) recomendaram a verificação de um índice de adequação geral do modelo, denominado GoF (Goodnessof Fit), obtido pela média geométrica entre o R2 médio (adequação do modelo estrutural) e a AVE média (adequação do modelo de mensuração). Um GoF mínimo de 0,36 é o recomendado como sendo adequado para estudos desenvolvidos nas áreas de ciências sociais e comportamentais (Wetzels, Odekerken-Schröder, \&Oppen, 2009).

\section{Resultados}

\subsection{Caracterização da amostra}

A amostra final foi composta por 416 consumidores. A tabela 1 apresenta os valores da caracterização da amostra.

Tabela 1 - Caracterização da amostra

\begin{tabular}{llcc}
\hline & & $\mathbf{n}$ & \% \\
\hline Gênero & Masculino & 109 & 26,2 \\
\multirow{3}{*}{ Idade } & Feminino & 307 & 73,8 \\
& Até 18 anos & 3 & 0,7 \\
& Entre 18 e 25 anos & 36 & 8,7 \\
& Entre 26 e 29 anos & 174 & 41,8 \\
& Entre 30 e 40 anos & 162 & 38,9 \\
\multirow{4}{*}{ Renda } & Acima de 40 anos & 41 & 9,9 \\
& Até $\mathrm{R} \$ 880,00$ & 3 & 0,7 \\
& Entre $\mathrm{R} \$ 880,00$ e $\mathrm{R} \$ 2.640,00$ & 30 & 7,2 \\
& Entre $\mathrm{R} \$ 2.640,00$ e $\mathrm{R} \$ 4.400,00$ & 329 & 79,1 \\
& Entre $\mathrm{R} \$ 4.400,00$ e $\mathrm{R} \$ 8.800,00$ & 46 & 11,1 \\
& Acima de $\mathrm{R} \$ 8.800,00$ & 8 & 1,9 \\
\hline
\end{tabular}

Fonte: Dados da pesquisa.

\subsection{Verificação dos pressupostos para MEE}

O teste de Levene indicou ausência de heterocedasticidade, apresentando indicadores não significativos, ao mesmo tempo em que os VIFs indicaram a ausência da multicolinearidade. Do mesmo modo, o teste $Z$ de KolmogorovSmirnov apresentou indicadores significativos, com o que se conclui que a amostra não é aderente a uma distribuição normal. Esse fato corrobora a decisão de utilização do método de mínimos quadrados parciais, pois modelos baseados na matriz de covariância, por caracterizarem-se como testes paramétricos, exigem que a distribuição seja normal.

\subsection{Análise do modelo de mensuração}

Múltiplos critérios devem ser empregados para avaliar o ajustamento do modelo teórico de modo geral (Bagozzi\& Yi, 1988; Hairet al., 2009).A validade convergente foi observada pelas cargas apresentadas. No 
modelo que utilizou os dados de toda a amostra, as cargas $\lambda$ variaram de 0,612 ( $\lambda$ COGN_5) até 0,834 ( $\left.\lambda R C \_04\right)$. A validade discriminante foi verificada pelo critério proposto por Fornell e Larcker (1981), constatando que as raízes quadradas de cada variável latente eram superiores à correlação entre elas e as demais variáveis latentes dos modelos, como demonstrado na tabela 2.

Tabela 2 - Análise da validade descriminante do modelo.

\begin{tabular}{lcccc}
\hline Construtos & Conf & ExpComp & Recompra & Satisf \\
\hline Conf & $\mathbf{0 , 7 7 6}$ & & & \\
ExpComp & 0,540 & $\mathbf{0 , 6 2 6 7}$ & & \\
Recompra & 0,444 & 0,731 & $\mathbf{0 , 7 8 6}$ & \\
Satisf & 0,538 & 0,674 & 0,616 & $\mathbf{0 , 8 0 3}$ \\
\hline
\end{tabular}

Fonte: Dados da pesquisa.

Os indicadores de ajuste dos modelos foram satisfatórios. A AVE foi,superior a0,50 em todos os casos, com exceção da ExpComp. A confiabilidade composta de todas as variáveis latentes foi superior a 0,7 e os coeficientes Alpha de Cronbach foram superiores ao limite mínimo. Além disso, os indicadores de ajustamento geral (GoF) foram superiores a 0,36 , podendo ser considerados como adequados. De modo geral, o ajustamento do modelo está razoável e pode ser aceito. A tabela 3 apresenta os indicadores identificados.

Tabela 3 - Índices de ajustamento do modelo.

\begin{tabular}{lcccc}
\hline \multirow{2}{*}{ Construtos } & \multicolumn{4}{c}{ GoF = 0,496 } \\
\cline { 2 - 5 } & AVE & CC & $\mathbf{R}^{\mathbf{2}}$ & $\mathbf{\alpha}$ \\
\hline Conf & 0,604 & 0,859 & 0,291 & 0,781 \\
ExpComp & 0,392 & 0,933 & & 0,925 \\
Recompra & 0,618 & 0,889 & 0,563 & 0,845 \\
Satisf & 0,645 & 0,900 & 0,454 & 0,862 \\
& $>0,5$ & $>0,6$ & & $>0,6$ \\
\hline
\end{tabular}

Nota. CC - Confiabilidade composta; $\alpha$ - Alpha de Cronbach; GoF - Goodnessof Fit.

Fonte: Dados da pesquisa.

\subsection{Análise do modelo estrutural e teste de hipóteses}

Na figura 2 podem ser observados os coeficientes de regressão associados à variável observada experiência de compra (ExpComp) e o quanto esta impacta nas variáveis latentes intenção de recompra (Recompra), satisfação (Satisf) e confiança (Conf).

A intenção de recompra (Recompra) possui o maior coeficiente de regressão $(0,575)$ com a variável experiência de compra (ExpComp), seguido de um coeficiente de regressão $(0,220)$ com a variável satisfação (Satis), e um coeficiente de regressão $(0,015)$ com a variável confiança (Conf). Portanto, quando a variável intenção de recompra (Recompra) aumenta de uma unidade, a maior contribuição para vem da experiência de compra (ExpComp), seguida pela satisfação (Satis).

Na figura 2 também podem ser observados os valores dos coeficientes de determinação da variância $\left(R^{2}\right)$ das variáveis dependentes: experiência de compra (ExpComp), intenção de recompra (Recompra), satisfação (Satisf) e confiança (Conf). Esses coeficientes indicam o percentual de variância da variável dependente, que é explicado pelas variáveis independentes. Os valores de $\mathrm{R}^{2}$ obtidos estão no interior dos círculos que representam essas variáveis. No caso da variável intenção de recompra (Recompra), o valor do coeficiente de determinação da variância $\left(r^{2}\right)$ obtido foi de $56,3 \%$. no caso da variável satisfação (Satisf), o valor do coeficiente de determinação da variância $\left(R^{2}\right)$ obtido foi de $45,5 \%$.Para a variável confiança (Conf), o coeficiente de determinação da variância $\left(R^{2}\right)$ obtido foi de $29,2 \%$.

Para a validação do modelo estrutural, foi utilizado o algoritmo de bootstrapping (amostragem aleatória) do software SmartPLS 2.0M3 (Ringleet al., 2005), com o parâmetro 5000 para o número de casos e amostras. 
A experiência de compra (ExpComp) tem relação positiva e significante com a intenção de recompra (Recompra). Consumidores que vivenciam experiências de compra positivas são mais propensos a repetirem essas experiências, realizando novas compras no estabelecimento $(\beta=0,575, t=10,362, p<0,001)$. Resta verificar se o efeito de mediação existe para que se possam comprovar as hipóteses inicialmente formuladas.

Para análise do efeito da mediação (ver tabela 4), a variável independente deve estar significantemente relacionada com a variável dependente e com as variáveis mediadoras (Baron \& Kenny, 1986). Para verificar se o efeito indireto da variável independente sobre a variável dependente é resultado da variável mediadora, empregou-se a abordagem analítica proposta por Hayes, Preacher e Myers (2011). A figura 2 mostra o efeito total da experiência de compra na intenção de recompra (c), sendo que o item b) expressa o efeito total da experiência de compra na intenção de recompra como a soma do efeito direto (c') e do efeito indireto $\left(a^{*} b\right)+\left(a^{\prime *} b^{\prime}\right)$.

Tabela 4 - Efeito nas variáveis endógenas

\begin{tabular}{|c|c|c|c|}
\hline Efeito na variável endógena & Efeito direto & t-value & Sig \\
\hline \multicolumn{4}{|l|}{ Satisfação $\left(\mathrm{R}^{2}=0,455\right)$} \\
\hline - Experiência de Compra (a) & 0,674 & 11,690 & $* * *$ \\
\hline \multicolumn{4}{|l|}{ Confiança $\left(R^{2}=0,292\right)$} \\
\hline - Experiência de Compra (a') & 0,540 & 7,561 & $* * *$ \\
\hline \multicolumn{4}{|l|}{ Recompra $\left(R^{2}=0,563\right)$} \\
\hline - $\mathrm{H}_{1}$ : Experiência de Compra (c') & 0,638 & 10,123 & $* * \star$ \\
\hline - Satisfação (b) & 0,220 & 4,252 & $* * *$ \\
\hline - Confianca (b') & 0,015 & 0,360 & n.s. \\
\hline
\end{tabular}

Nota. ${ }^{*} p<0,05 ;{ }^{* *} p<0,01 ;{ }^{* * *} p<0,001 ;$ n.s. $=$ não significante.

Fonte:Dados da pesquisa.

Com a satisfação e a confiança como mediadoras, o efeito experiência de compra na intenção de recompra diminui, mas ainda continua significante $(\beta=0,638, t=18,211, p<0,001)$, conforme mostrado na figura 2. Esses achados corroboram a ideia de que, para manterem a intenção de recompra de seus clientes, os varejistas precisam manter ou aumentar a experiência de compra de seus respectivos clientes, pois índices mais altos de experiência de compra levam a índices mais altos de intenção de recompra.

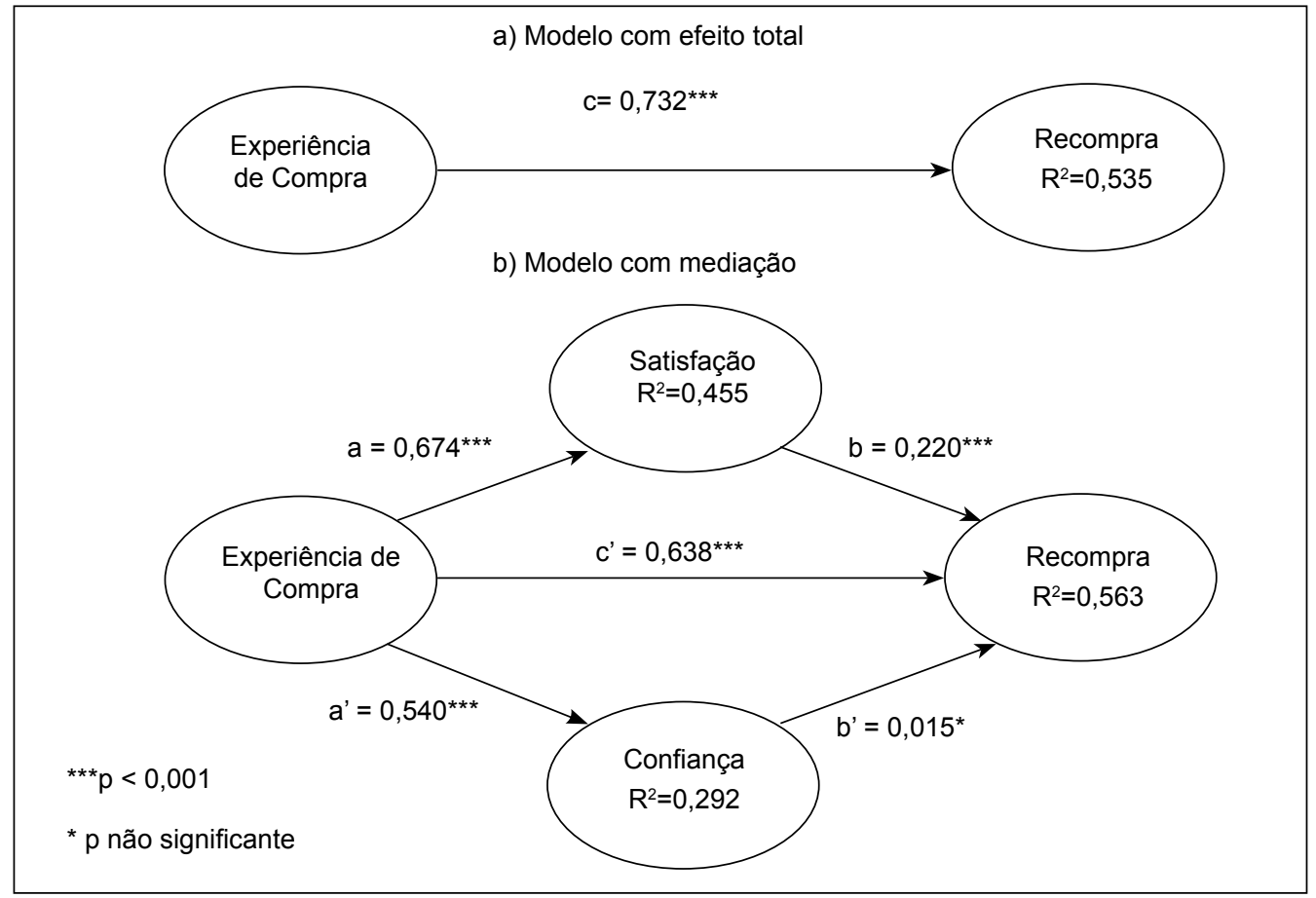

Figura 2 - Modelo estrutural e modelo com múltiplas mediações

Fonte: Dados da pesquisa.

O resumo dos testes de mediação pode ser observado na tabela 5. 
Tabela 5 - Resumo dos testes de mediação

\begin{tabular}{|c|c|c|c|c|c|}
\hline \multicolumn{2}{|c|}{$\begin{array}{l}\text { Efeito total da Experiência } \\
\text { de Compra na Recompra (c) }\end{array}$} & \multicolumn{2}{|c|}{$\begin{array}{l}\text { Efeito direto da Experiência } \\
\text { de Compra na Recompra }\end{array}$} & \multicolumn{2}{|c|}{$\begin{array}{c}\text { Efeito indireto da Experiência de } \\
\text { Compra na Recompra }\end{array}$} \\
\hline Coeficiente & $t$ value & Coeficiente & $t$ value & Ponto estimado & Efeito \\
\hline 0,732 & 14,142 & $\mathrm{H}_{1} \mathrm{c}=0,638$ & 10,123 & $\begin{array}{c}\text { Total }=a^{*} b+a^{\prime *} b^{\prime} \\
\mathrm{H}_{2} \text { a*b (via Satisfação) } \mathrm{H}_{3} \mathrm{a}^{\prime *} \mathrm{~b}^{\prime} \text { (via Confiança) }\end{array}$ & $\begin{array}{c}0,15 \\
0,14 \\
0,008\end{array}$ \\
\hline
\end{tabular}

Fonte: Dados da pesquisa.

Para verificar se o efeito indireto da variável independente sobre a variável dependente é resultado da variável mediadora, realizam-se os testes de Sobel, Aroian e Goodman (Baron \& Kenny, 1986). A tabela 6 apresenta os resultados para os testes de mediação.

Tabela 6 - Testes de Sobel, Aroian e Goodman

\begin{tabular}{llcccccc}
\hline & Caminho & Sobel & p-value & Aroian & p-value & Goodman & p-value \\
\hline $\mathrm{H}_{1}(\mathrm{NR})$ & ExpCom $\rightarrow$ Satis $\rightarrow$ Recompra & 4,1082 & $* * *$ & 4,095 & $* * *$ & 4,121 & $* * *$ \\
$\mathrm{H}_{2}(\mathrm{R})$ & ExpCom $\rightarrow$ Conf $\rightarrow$ Recompra & 0,3554 & n.s & 0,3524 & n.s & 0,3584 & n.s. \\
\hline
\end{tabular}

Nota: ${ }^{*} p<0,05 ;{ }^{* *} p<0,01 ;{ }^{* * *} p<0,001 ; n . s .=$ não significante; $(R)=$ Hipótese rejeitada; $(N R)=$ Hipótese não rejeitada

Fonte:Dados da pesquisa.

Os resultados mostram que existe uma mediação positiva e significante para os três testes quando se considera a variável satisfação. Entretanto, o mesmo não ocorre com a variável mediadora confiança, pois, para os três testes, os resultados foram não significativos.

\section{Discussão}

Recuperando o objetivo inicial proposto, buscou-se investigar a relação entre a experiência de compra e a intenção de recompra e o papel mediador da satisfação e da confiança do consumidor nessa relação, testando-os em modelagem de equações. Os resultados do estudo mostraram evidências dos antecedentes da intenção de recompra, seus impactos e seus intra-relacionamentos. Os testes de hipóteses apontaram para a existência da relação de mediação pela satisfação, mas não pela confiança, indicando que o modelo possui uma boa adequação.

A satisfação teve papel de mediação entre a experiência de compra do consumidor e a intenção de recompra.Entende-se aqui que quanto mais experiência de compra o consumidor acumular maior será sua satisfação e mais intenção de retornar e repetir a experiência.

A confiança, por sua vez, não apresentou papel de mediação.Nota-se que existe uma relação entra a experiência de compra e a confiança, e a explicação para isso reside no fato de que quanto mais experiência de compra o consumidor acumula, mais confiante ele fica no processo. Porém, não significa que a confiança acumulada pelo consumidor se reflita na intenção de retornar e repetir a experiência de compra. Este papel é executado pela função de mediação da satisfação.

Os resultados satisfazem parcialmente o que já foi encontrado em outros trabalhos empíricos, quando se investigou a relação mediada pela satisfação (Julander \& Söderlund, 2003; Mittal \& Kamakura, 2001; Rose, Clark, Samouel, \& Hair, 2012; Zboja \& Voorhees, 2006). Por exemplo, nos resultados relatados por de Jin e Park (2006), as fontes da confiança mudam conforme as experiências de compras aumentam e a satisfação do consumidor permanece inalterada, apesar das experiências de compra. No mesmo sentido, o trabalho de Zboja e Voorhees (2006) mostrou que a confiança e a satisfação possuem uma relação positiva e significante com a intenção de recompra.

Outro ponto importante que merece uma discussão é o total da variância da intenção de recompra explicada pelas variáveis antecessoras. A experiência de compra explica $53 \%$ da variância da intenção de recompra quando se considera o efeito direto dessa variável antecessora. Entretanto, as variáveis satisfação e confiança possuem apenas uma pequena porção explicativa da variância da intenção de recompra, 
considerando-se apenas a mediação $2,8 \%$ de contribuição na variância explicada. Como temos no total $56 \%$ da variância da intenção de recompra explicada pelas três variáveis antecessoras (experiência de compra, satisfação e confiança), o restante é explicado por outras variáveis que não foram objeto desta pesquisa.

Teoricamente, o trabalho contribui para a área de Marketing e de Administração, trazendo a discussão do papel de mediação da satisfação no processo de experiência de compra e intenção de recompra pelo consumidor. Este achado confirma outros trabalhos empíricos desenvolvidos por pesquisadores da área (Mittal \& Kamakura, 2001; Zboja \& Voorhees, 2006). Outro resultado importante para a pesquisa em Marketing foi mostrar que a experiência de compra é um construto complexo e multifacetado, formado por diversos outros construtos. Neste trabalho, foram escolhidas as dimensões cognição, afeto, social e atmosfera da loja (Frow \& Payne, 2007; Haytko \& Baker, 2004; Luo, 2005; White \& Dahl, 2006).Entretanto,é importante destacar que outras dimensões fazem parte do construto, como alegria (Jin \& Sternquist, 2004), humor (Holbrook \& Hirschman, 1982), diferenciação (Schmitt, 1999), entre outros.

Gerencialmente, os resultados do modelo estrutural e dos testes de mediação possuem implicações para o setor de varejo de forma geral. Especificamente, os resultados fornecem novos insights para os gestores das organizações no desenvolvimento de estratégias internas. Quando desenvolverem suas respectivas estratégias, gestores de varejo deverão considerar que a experiência de compra que seus respectivos clientes vivenciam em suas lojas afetará diretamente seu nível de satisfação e que, por sua vez,está relacionado diretamente com a intenção de retornar e repetir a experiência de compra. Esses resultados oferecem uma grande oportunidade para os varejistas, como o potencial incremento nas vendas pela criação de ambientes que conduzam a uma experiência de compra positiva.

Embora não eliminem a contribuição para a academia, algumas limitações deste trabalho devem ser destacadas para que possam ser superadas em estudos futuros. Primeiro, este estudo tem um caráter transversal. A abordagem se baseia em uma análise de um único momento no tempo. Segundo, por conveniência, foram escolhidos consumidores do varejo de roupas como objeto de investigação.Essa escolha limita os achados deste trabalho ao contexto investigado. Terceiro, os resultados apontaram que a confiança não é mediadora na relação entre a experiência de compra e a intenção de recompra, em oposição ao que aponta a literatura sobre o tema. A quarta limitação refere-se ao emprego, nesta pesquisa, da combinação de escalas desenvolvidas por vários pesquisadores, com base em dimensões por eles escolhidas para mensurar a experiência de compra.

\section{Conclusão}

Esta pesquisa desenvolveu e testou empiricamente um modelo que representa as relações entre a intenção de recompra e a experiência de compra do cliente, tendo como mediação a satisfação e contribui para a literatura acadêmica pela apresentação de evidências empíricas dessa relação.Os resultados encontrados sugerem que o nível de experiência do cliente afeta positivamente sua intenção de repetir a compra e a satisfação possui um papel de mediação nessa relação. Não é possível identificar efeito de mediação para a variável confiança nos resultados.

Futuros estudos devem explorar um caráter longitudinal, que poderia trazer novas descobertas e fazer avançar o conhecimento sobre a temática. É altamente desejável que outros setores (varejo de calçados, ou alimentos, bares e restaurantes entre outros) possam ser investigados. Seria importante que este estudo fosse reproduzido, com o intuito de levantar mais informações sobre os resultados encontrados para a mediação da confiança. Seria desejável que uma escala que refletisse a realidade e a personalidade do consumidor brasileiro fosse desenvolvida e testada para futuras aplicações, baseada não só nas dimensões escolhidas neste trabalho, mas também em outras dimensões que pudessem refletir com mais precisão o fenômeno investigado.

\section{Referências}

Alves, C. A. (2016). Confiança e comprometimento e sua relação com o desempenho e a intenção de recompra do varejista dentro do canal de marketing. Revista Ciências Administrativas, 22(1), 100-129. 
Alves, C. A. (2017). Estou Satisfeito: Um Estudo sobre a Lealdade do Consumidor em Restaurantes Empregando a Escala DINESERV. REMark, 16(3), 334-350.

Anderson, J. C., \& Gerbing, D. W. (1988). Structural Equation Modeling in Practice: A Review and Recommended Two-Step Approach. Psychological Bulletin, 103(3), 411-423.

Athiyaman, A. (2004). Antecedents and consequences of student satisfaction with university services: a longitudinal analysis. Academy of Marketing Studies Journal, 8(1), 89-105.

Babin, B. J., Darden, W. R., \& Babin, L. A. (1998). Negative emotions in marketing research: affect or artifact? Journal of Business Research, 42(3), 271-285.

Bagdare, S., \& Jain, R. (2013). Measuring retail customer experience. International Journal of Retail \& Distribution Management, 41(10), 790-804.

Bagozzi, R. P., Gopinath, M., \& Nyer, P. U. (1999). The role of emotions in marketing. Journal of the Academy of Marketing Science, 27(2), 184-206.

Bagozzi, R. P., \& Yi, Y. (1988). On the evaluation of structural equation models. Journal of the Academy of Marketing Science, 16(1), 74-94.

Bagozzi, R. P. (1980). Causal models in marketing. New York: John Wiley \& Sons Inc.

Baker, J., Grewal, D., \& Parasuraman, A. (1994). The influence of store environment on quality inferences and store image. Journal of the Academy of Marketing Science, 22(4), 328-339.

Ballantine, P. W., Jack, R., \& Parsons, A. G. (2010). Atmospheric cues and their effect on the hedonic retail experience. International Journal of Retail \& Distribution Management, 38(8), 641-653.

Baron, R. M., \& Kenny, D. A. (1986). The moderator-mediator variable distinction in social psychological research: Conceptual, strategic, and statistical considerations. Journal of Personality and Social Psychology, 51(6), 1173.

Berry, L. L., \& Carbone, L. P. (2007). Build loyalty through experience management. Quality Progress, 40(9), 26-32.

Bido, D. de S., Godoy, A. S., Ferreira, J. F., Kenski, J. M., \& Scartezini, V. N. (2011). Examinando a relação entre aprendizagem individual, grupal e organizacional em uma instituição financeira. Revista Eletrônica de Administração, 17(1), 58-85.

Borges, A., Chebat, J.-C., \& Babin, B. J. (2010). Does a companion always enhance the shopping experience? Journal of Retailing and Consumer Services, 17(4), 294-299.

Cacioppo, J. T., \& Petty, R. E. (1982). The need for cognition. Journal of Personality and Social Psychology, 42(1), 116.

Cardozo, R. N. (1965). An experimental study of customer effort, expectation, and satisfaction. Journal of Marketing Research, 2(3), 244-249.

Chebat, J.-C., Chebat, C. G., \& Vaillant, D. (2001). Environmental background music and in-store selling. Journal of Business Research, 54(2), 115-123.

Chin, W. W., \& Newsted, P. R. (1999). Structural equation modelling analysis with small samples using partial least squares. In R. H. Hoyle (Ed.), Statistical strategies for small sample research. Thousand Oaks, CA: Sage.

Cronbach, L. J. (1971). Test Validation. In R. Thorndike (Ed.), Educational Measurement (2nd ed., p. 443). Washington, DC: American Council on Education.

Entenberg, R. D. (1960). Suggested changes in census classifications of retail trade. The Journal of Marketing, 24(3), 39-43. 
Feijó, F. R., \& Botelho, D. (2012). Efeito dos fatores de merchandising nas vendas do varejo. RAE-Revista de Administração de Empresas, 52(6), 628-642.

Flanagan, P., Johnston, R., \& Talbot, D. (2005). Customer confidence: The development of a "pre-experience" concept. International Journal of Service Industry Management, 16(4), 373-384.

Fornell, C., \& Larcker, D. F. (1981). Evaluating structural equation models with unobservable variables and measure. Journal of Marketing Research, 28(4), 39-50.

Frow, P., \& Payne, A. (2007). Towards the "perfect"customer experience. Journal of Brand Management, 15(2), 89-101.

Fullerton, G. (2005). The impact of brand commitment on loyalty to retail service brands. Canadian Journal of Administrative Sciences/Revue Canadienne Des Sciences de l'Administration, 22(2), 97-110.

Garbarino, E., \& Johnson, M. S. (1999). The different roles of satisfaction, trust, and commitment in customer relationships. The Journal of Marketing, 63(2), 70-87.

Gentile, C., Spiller, N., \& Noci, G. (2007). How to sustain the customer experience:: An overview of experience components that co-create value with the customer. European Management Journal, 25(5), 395-410.

Goby, V. P. (2006). Personality and online/offline choices: MBTI profiles and favored communication modes in a Singapore study. CyberPsychology \& Behavior, 9(1), 5-13.

Goswami, P., \& Mishra, M. S. (2009). Would Indian consumers move from kirana stores to organized retailers when shopping for groceries? Asia Pacific Journal of Marketing and Logistics, 21(1), 127-143.

Grace, D., \& O'Cass, A. (2005). An examination of the antecedents of repatronage intentions across different retail store formats. Journal of Retailing and Consumer Services, 12(4), 227-243.

Grewal, D., Baker, J., Levy, M., \& Voss, G. B. (2003). The effects of wait expectations and store atmosphere evaluations on patronage intentions in service-intensive retail stores. Journal of Retailing, 79(4), 259-268.

Hair, J. F., Black, W. C., Babin, B. j., Anderson, R. E., \& Tatham, R. L. (2009). Análise multivariada de dados. Porto Alegre: Bookman.

Havlena, W. J., \& Holbrook, M. B. (1986). The varieties of consumption experience: comparing two typologies of emotion in consumer behavior. Journal of Consumer Research, 13(3), 394-404.

Hayes, A. F., Preacher, K. J., \& Myers, T. A. (2011). Mediation and the estimation of indirect effects in political communication research. In E. P. Bucy \& R. L. Holbert (Eds.), Sourcebook for political communication research: Methods, measures, and analytical techniques (pp. 434-465). New York: Routledge.

Haytko, D. L., \& Baker, J. (2004). It's all at the mall: exploring adolescent girls' experiences. Journal of Retailing, 80(1), 67-83.

Hellier, P. K., Geursen, G. M., Carr, R. A., \& Rickard, J. A. (2003). Customer repurchase intention: A general structural equation model. European Journal of Marketing, 37(11/12), 1762-1800.

Herzog, R., \& Bachman, J. G. (1981). Effects of Questionnaire Length on Response Quality. Public Opinion Quartely, 45(4), 549-599.

Holbrook, M. B. (1986). Emotion in the consumption experience: toward a new model of the human consumer. In R. A. Peterson, W. D. Hoyer, \& W. Wilson (Eds.), The role of affect in consumer behavior: Emerging theories and applications (pp. 17-52). Lexington: MA: Health.

Holbrook, M. B., \& Hirschman, E. C. (1982). The experiential aspects of consumption: Consumer fantasies, feelings, and fun. Journal of Consumer Research, 9(2), 132-140.

Janda, S., \& Ybarra, A. (2005). Do product and consumer characteristics affect the relationship between online experience and customer satisfaction? Journal of Internet Commerce, 4(4), 133-151. 
Jin, B., \& Park, J. Y. (2006). The moderating effect of online purchase experience on the evaluation of online store attributes and the subsequent impact on market response outcomes. Advances in Consumer Research, 33(1), 203-211.

Jin, B., \& Sternquist, B. (2004). Shopping is truly a joy. The Service Industries Journal, 24(6), 1-18.

Johnson, D., \& Grayson, K. (2005). Cognitive and affective trust in service relationships. Journal of Business Research, 58(4), 500-507.

Johnson, A. R., \& Stewart, D. W. (2005). A reappraisal of the role of emotion in consumer behavior. In N. K. Malhotra (Ed.), Review of Marketing Research (Vol. 1, pp. 3-33). Armonk, NY: ME Sharpe.

Johnson-George, C., \& Swap, W. C. (1982). Measurement of specific interpersonal trust: Construction and validation of a scale to assess trust in a specific other. Journal of Personality and Social Psychology, 43(6), 1306.

Johnson, M. D., Herrmann, A., \& Huber, F. (2006). The evolution of loyalty intentions. Journal of Marketing, 70(2), 122-132.

Johnston, R., \& Kong, X. (2011). The customer experience: a road-map for improvement. Managing Service Quality: An International Journal, 21(1), 5-24.

Joreskog, K. G., \& Sorbom, D. (1996). LISREL 8: User's Reference Guide. Chicago: Scientific Software International.

Julander, C., \& Söderlund, M. (2003). Effects of switching barriers on satisfaction, repurchase intentions and attitudinal loyalty. (SSE/EFI Working Paper Series in Business Administration, No. 2003:1). Stockholm:Stockholm School of Economics.

Kesari, B., \& Atulkar, S. (2016). Satisfaction of mall shoppers: A study on perceived utilitarian and hedonic shopping values. Journal of Retailing and Consumer services, 31(4), 22-31.

Kiecker, P., \& Hartman, C. L. (1994). Predicting Buyers' Selection of Interpersonal Sources: The Role of Strong Ties and Weak Ties. Advances in Consumer Research, 21(1), 464-469.

Kim, C., Galliers, R. D., Shin, N., Ryoo, J.-H., \& Kim, J. (2012). Factors influencing Internet shopping value and customer repurchase intention. Electronic Commerce Research and Applications, 11(4), 374-387.

Kline, R. B. (2005). Principles and Practice of Structural Equation Modeling. (D. A. Kenny, Ed.) ( $\left.2^{\circ}\right)$. New York: The Guilford Press.

Krzanowski, W. J. (2007). Statistical principles and techniques in scientific and social investigations. London: Oxford University Press.

Law, D., Wong, C., \& Yip, J. (2012). How does visual merchandising affect consumer affective response? An intimate apparel experience. European Journal of Marketing, 46(1/2), 112-133.

Lemke, F., Clark, M., \& Wilson, H. (2011). Customer experience quality: an exploration in business and consumer contexts using repertory grid technique. Journal of the Academy of Marketing Science, 39(6), 846-869.

Levin, J., \& Fox, A. (2006). Estatítica para as Ciências Humanas. São Paulo: Pearson.

Lindsey-Mullikin, J., \& Munger, J. L. (2011). Companion shoppers and the consumer shopping experience. Journal of Relationship Marketing, 10(1), 7-27.

Lopes, H. E. G. (2008). Abrindo a caixa preta: considerações sobre a utilização da Análise Fatorial Confirmatória nas pesquisas em Administração. Revista Economia \& Gestão, 5(11), 97-116.

Luo, X. (2005). How does shopping with others influence impulsive purchasing? Journal of Consumer Psychology, 15(4), 288-294.

Malhotra, N. K. (2011). Pesquisa de Marketing. Uma Orientação Aplicada (6º. Porto Alegre: Bookman. 
Matzler, K., Faullant, R., Renzl, B., \& Leiter, V. (2005). The relationship between personality traits (extraversion and neuroticism), emotions and customer self-satisfaction. Innovative Marketing, 1(2), 32-39.

Mehrabian, A., \& Russell, J. A. (1974). An approach to environmental psychology. Massachusetts: The MIT Press.

Meyer, C., \& Schwager, A. (2007). Understanding customer experience. Harvard Business Review, 85(2), 116-126.

Michon, R., Chebat, J.-C., \& Turley, L. W. (2005). Mall atmospherics: the interaction effects of the mall environment on shopping behavior. Journal of Business Research, 58(5), 576-583.

Mittal, V., \& Kamakura, W. A. (2001). Satisfaction, repurchase intent, and repurchase behavior: Investigating the moderating effect of customer characteristics. Journal of Marketing Research, 38(1), 131-142.

Mittal, V., Ross, W. T., \& Baldasare, P. M. (1998). The asymmetric impact of negative and positive attributelevel performance on overall satisfaction and repurchase intentions. Journal of Marketing, 62(1), 33-47.

Mohan, G., Sivakumaran, B., \& Sharma, P. (2013). Impact of store environment on impulse buying behavior. European Journal of Marketing, 47(10), 1711-1732.

Moorman, C., Zaltman, G., \& Deshpande, R. (1992). Relationships between providers and users of marketing research: The dynamics of trust between and within organisations. Journal of Marketing Research, 29(3), 314-329.

Moorman, C., Deshpande, R., \& Zaltman, G. (1993). Factors affecting trust in market research relationships. The Journal of Marketing, 57(1), 81-101.

Morgan, R. M., \& Hunt, S. D. (1994). The commitment-trust theory of relationship marketing. Journal of Marketing, 58(3), 20-38.

Morrin, M., \& Ratneshwar, S. (2000). The impact of ambient scent on evaluation, attention, and memory for familiar and unfamiliar brands. Journal of Business Research, 49(2), 157-165.

Mower, J. M., Kim, M., \& Childs, M. L. (2012). Exterior atmospherics and consumer behavior: influence of landscaping and window display. Journal of Fashion Marketing and Management: An International Journal, 16(4), 442-453.

Nicholls, J. A. F. (1997). Time and companionship: key factors in Hispanic shopping behavior. Journal of Consumer Marketing, 14(3), 194-205.

Oliver, R. L. (1980). A cognitive model of the antecedents and consequences of satisfaction decisions. Journal of Maketing Research, 17(4), 460-469.

Preacher, K. J., Rucker, D. D., \& Hayes, A. F. (2007). Addressing moderated mediation hypotheses: Theory, methods, and prescriptions. Multivariate Behavioral Research, 42(1), 185-227.

Pullman, M. E., \& Gross, M. A. (2004). Ability of experience design elements to elicit emotions and loyalty behaviors. Decision Sciences, 35(3), 551-578.

Rawson, A., Duncan, E., \& Jones, C. (2013). The truth about customer experience. Harvard Business Review, 91(9), 90-98.

Rempel, J. K., Holmes, J. G., \&Zanna, M. P. (1985). Trust in close relationships. Journal of personality and social psychology, 49(1), 95-112.

Ringle, C. M., Wende, S., \& Will, A. (2005). SmartPLS 2.0 M3 (beta). Hamburg: University of Hamburg.

Rose, S., Clark, M., Samouel, P., \& Hair, N. (2012). Online customer experience in e-retailing: an empirical model of antecedents and outcomes. Journal of Retailing, 88(2), 308-322.

Schmitt, B. (1999). Experiential marketing. Journal of Marketing Management, 15(1-3), 53-67. 
Stefanini, C. J., Alves, C. A., \& Marques, R. B. (2018). Vamos almoçar? Um estudo da relação hospitalidade, qualidade em serviços e marketing de experiência na satisfação dos clientes de restaurantes. Revista Brasileira de Pesquisa em Turismo, 12(1), 57-79.

Stefanini, C. J., Yamashita, A. P., Alves, C. A., \& Marques, R. B. (2017). Factors related to perception of quality in hosting services among business tourists from São Paulo/Brazil. Revista Espacios, 38(57), 20-30.

Tauber, E. M. (1972). Why do people shop? The Journal of Marketing, 36(4), 46-49.

Teixeira, J. M., \& Hernandez, J. D. C. (2012). Valores de compra hedônico e utilitário: os antecedentes e as relações com os resultados do varejo. Revista Eletrônica de Administração, Porto Alegre, 71(1), 130-160.

Tenenhaus, M., Vinzi, V. E., Chatelin, Y.-M., \& Lauro, C. (2005). PLS path modeling. Computational Statistics \& Data Analysis, 48(1), 159-205.

Verhoef, P. C., Franses, P. H., \& Hoekstra, J. C. (2002). The effect of relational constructs on customer referrals and number of services purchased from a multiservice provider: does age of relationship matter? Journal of the Academy of Marketing Science, 30(3), 202-216.

Verhoef, P. C., Lemon, K. N., Parasuraman, A., Roggeveen, A., Tsiros, M., \& Schlesinger, L. A. (2009). Customer experience creation: Determinants, dynamics and management strategies. Journal of Retailing, 85(1), 31-41.

Voss, C. A. (2003). Rethinking paradigms of service: Service in a virtual environment. International Journal of Operations \& Production Management, 23(1), 88-104.

Wetzels, M., Odekerken-Schröder, G., \& Oppen, C. van. (2009). Using PLS path modeling for assessing hierarchical construct models guidelines and empirical illustration, MIS quarterly, 33(1), 177-195.

White, K., \& Dahl, D. W. (2006). To be or not be? The influence of dissociative reference groups on consumer preferences. Journal of Consumer Psychology, 16(4), 404-414.

Yalch, R. F., \& Spangenberg, E. R. (2000). The effects of music in a retail setting on real and perceived shopping times. Journal of Business Research, 49(2), 139-147.

Zboja, J. J., \& Voorhees, C. M. (2006). The impact of brand trust and satisfaction on retailer repurchase intentions. Journal of Services Marketing, 20(6), 381-390.

Zeithaml, V. A., Berry, L. L., \& Parasuraman, A. (1996). The behavioral consequences of service quality. The Journal of Marketing, 60(2), 31-46.

Submetido em: 13/04/17

Aprovado em: 06/03/18 